\title{
Article \\ Effect of Point-Spread Function Reconstruction for Indeterminate PSMA-RADS-3A Lesions on PSMA-Targeted PET Imaging of Men with Prostate Cancer
}

\author{
Wajahat Khatri ${ }^{1,+}{ }^{(D)}$, Hyun Woo Chung ${ }^{2,+}+$, Rudolf A. Werner ${ }^{3}$, Jeffrey P. Leal ${ }^{1}$, Kenneth J. Pienta ${ }^{4}$, \\ Martin A. Lodge ${ }^{1} \mathbb{D}$, Michael A. Gorin ${ }^{5,6}$, Martin G. Pomper ${ }^{1,4}$ and Steven P. Rowe ${ }^{1,4, * \mathbb{C}}$ \\ 1 The Russell H. Morgan Department of Radiology and Radiological Science, Johns Hopkins University \\ School of Medicine, Baltimore, MD 21287, USA; wkhatri1@jhmi.edu (W.K.); jleal1@jhmi.edu (J.P.L.); \\ mlodge1@jhmi.edu (M.A.L.); mpomper@jhmi.edu (M.G.P.) \\ 2 Department of Nuclear Medicine, Konkuk University Medical Center, Konkuk University School of Medicine, \\ Seoul 05030, Korea; 20080193@kuh.ac.kr \\ 3 Department of Nuclear Medicine, University Hospital Würzburg, 97080 Würzburg, Germany; \\ Werner_R1@ukw.de \\ 4 The James Buchanan Brady Urological Institute and Department of Urology, Johns Hopkins University \\ School of Medicine, Baltimore, MD 21287, USA; kpienta1@jhmi.edu \\ check for
updates \\ Citation: Khatri, W.; Chung, H.W.; \\ Werner, R.A.; Leal, J.P.; Pienta, K.J.; \\ Lodge, M.A.; Gorin, M.A.; Pomper, \\ M.G.; Rowe, S.P. Effect of \\ 5 Department of Urology, University of Pittsburgh School of Medicine, Pittsburgh, PA 15232, USA; \\ michaelagorin@gmail.com \\ 6 Urology Associates and UPMC Western Maryland, Cumberland, MD 21502, USA \\ * Correspondence: srowe8@jhmi.edu \\ + These authors contributed equally.
}

Point-Spread Function Reconstruction for Indeterminate PSMA-RADS-3A Lesions on PSMA-Targeted PET Imaging of Men with Prostate Cancer. Diagnostics 2021, 11, 665. https://doi.org/10.3390/ diagnostics11040665

Academic Editor: Rachel Bar-Shalom

Received: 22 February 2021

Accepted: 5 April 2021

Published: 7 April 2021

Publisher's Note: MDPI stays neutral with regard to jurisdictional claims in published maps and institutional affiliations.

Copyright: (c) 2021 by the authors Licensee MDPI, Basel, Switzerland. This article is an open access article distributed under the terms and conditions of the Creative Commons Attribution (CC BY) license (https:// creativecommons.org/licenses/by/ $4.0 /)$.

\begin{abstract}
Purpose: Prostate-specific membrane antigen (PSMA) positron emission tomography (PET) is emerging as an important modality for imaging patients with prostate cancer (PCa). As with any imaging modality, indeterminate findings will arise. The PSMA reporting and data system (PSMARADS) version 1.0 codifies indeterminate soft tissue findings with the PSMA-RADS-3A moniker. We investigated the role of point-spread function (PSF) reconstructions on categorization of PSMARADS-3A lesions. Methods: This was a post hoc analysis of an institutional review board approved prospective trial. Around $60 \mathrm{~min}$ after the administration of $333 \mathrm{MBq}(9 \mathrm{mCi}$ ) of PSMA-targeted ${ }^{18}$ F-DCFPyL, patients underwent PET/computed tomography (CT) acquisitions from the mid-thighs to the skull vertex. The PET data were reconstructed with and without PSF. Scans were categorized according to PSMA-RADS version 1.0, and all PSMA-RADS-3A lesions on non-PSF images were reevaluated to determine if any could be re-categorized as PSMA-RADS-4. The maximum standardized uptake values (SUVs) of the lesions, mean SUVs of blood pool, and the ratios of those values were determined. Results: A total of 171 PSMA-RADS-3A lesions were identified in 30 patients for whom both PSF reconstructions and cross-sectional imaging follow-up were available. A total of 13/171 (7.6\%) were re-categorized as PSMA-RADS-4 lesions with PSF reconstructions. A total of 112/171 $(65.5 \%)$ were found on follow-up to be true positive for PCa, with all 13 of the re-categorized lesions being true positive on follow-up. The lesions that were re-categorized trended towards having higher $\mathrm{SUV}_{\text {max }}$-lesion and SUV $\mathrm{Sax}_{\text {-lesion/SUV }}$ mean-blood-pool metrics, although these relationships were not statistically significant. Conclusions: The use of PSF reconstructions for ${ }^{18} \mathrm{~F}$-DCFPyL PET can allow the appropriate re-categorization of a small number of indeterminate PSMA-RADS-3A soft tissue lesions as more definitive PSMA-RADS-4 lesions. The routine use of PSF reconstructions for PSMA-targeted PET may be of value at those sites that utilize this technology.
\end{abstract}

Keywords: prostate-specific membrane antigen; reporting and data system; positron emission tomography 


\section{Introduction}

Prostate cancer ( $\mathrm{PCa})$ is the most common noncutaneous malignancy in men as well as the second most common cause of cancer death in men in the U.S.A. [1]. The high incidence of PCa has been a driving force for the development of new imaging techniques to improve staging and re-staging. Although conventional imaging with computed tomography (CT) and bone scan can adequately stage patients with widespread metastatic disease, the introduction of radiopharmaceuticals that bind the prostate-specific membrane antigen (PSMA), for targeted positron emission tomography/computed tomography (PET/CT), has facilitated the detection of small-volume lesions that can significantly alter treatment planning [2].

PSMA is a class II transmembrane glycoprotein that is a valuable target for imaging and therapy, as it is significantly overexpressed on malignant prostate cells [3]. Among the emerging indications for PSMA PET is the evaluation of patients with biochemical recurrence (BCR) after initial curative-intent therapy [4] and the identification of men with oligometastatic PCa (typically being defined as $\leq 5$ sites of distant disease) [5]. Sensitive lesion detection in men with BCR or oligometastatic disease is desired for directing further treatment, which might include targeted radiotherapy or surgery [6].

Given the increased use of such therapies for limited volume recurrent/metastatic disease, the confident characterization of lesions on PSMA PET is an important clinical need. For this reason, we developed the PSMA-RADS version 1.0 scoring system, which includes indeterminate categories for lesions that cannot be definitively ascertained to be PCa [7-10]. One such category is PSMA-RADS-3A, which describes lymph nodes and other soft tissue lesions that would be in the typical distribution of PCa but are small and have low-level uptake. We have previously found that approximately 75\% of PSMA-RADS-3A lesions will manifest as true positive sites of PCa on follow-up imaging [9]. Improvements in the evaluation of these small and generally subtle lesions so that they could be more definitively assigned as benign or malignant findings would be of value to guide accurate therapy planning.

Modern image reconstruction techniques, including point-spread function (PSF) reconstruction, can lead to improved signal-to-noise ratio and conspicuity of small or subtle lesions [11]. Hence, the aim of this study was to evaluate if PSF reconstructions for ${ }^{18} \mathrm{~F}-$ DCFPyL PET/CT affect the characterization of PSMA-RADS-3A lesions.

\section{Materials and Methods}

\subsection{Patient Population}

Patients with a history of pathologically diagnosed PCa who had undergone an ${ }^{18} \mathrm{~F}$ DCFPyL PET/CT scan on an institutional review board approved prospective research protocol (ClinicalTrials.gov (accessed on 1 January 2021) NCT02825875) were screened as part of this post hoc analysis to identify patients with at least one PSMA-RADS-3A lesion. All patients had signed written, informed consent to participate in the original research trial. Patients were imaged under the auspices of a US Food and Drug Administration Investigational New Drug Application (IND 121064). Clinical and demographic information, including age, serum prostate-specific antigen (PSA) levels, and PCa treatment history, was collected.

\subsection{Image Acquisition}

The radiosynthesis of ${ }^{18} \mathrm{~F}-\mathrm{DCFPyL}$ was performed as previously described [12]. Images were acquired in a manner consistent with prior studies [13]. In brief, all patients were asked to refrain from eating or drinking (other than water and medications) for at least $4 \mathrm{~h}$ before the intravenous injection of approximately $333 \mathrm{MBq}(9 \mathrm{mCi})$ of ${ }^{18} \mathrm{~F}-\mathrm{DCFPyL}$. One hour after the injection, whole-body PET/CT was performed (from the midthighs to the vertex of the skull) on one of two clinical scanners. Patients included in the final analysis were imaged on a 128-slice Biograph mCT scanner (Siemens) and reconstructed with both a standard ordered subset expectation maximization (OSEM) algorithm and OSEM with PSF. 
The standard reconstruction algorithm involved 3D OSEM with time-of-flight, 2 iterations, 21 subsets, and a $5 \mathrm{~mm}$ Gaussian filter. The higher spatial resolution PSF reconstruction involved 3D OSEM with time-of-flight and PSF modelling, 2 iterations, 21 subsets, and no post-reconstruction filter. CT (40 effective $\mathrm{mAs}$ ) was utilized for attenuation correction.

\subsection{Image Analysis}

${ }^{18}$ F-DCFPyl PET/CT scans reconstructed with OSEM but without PSF were centrally reviewed by an experienced reader (HWC), and lesions were characterized according to PSMA-RADS version 1.0. A second experienced reader (SPR) verified the lesion characterizations on OSEM without PSF and then re-characterized the PSMA-RADS-3A lesions with OSEM with PSF. As defined in the original description of the PSMA-RADS 1.0 scoring system, the central reviewers considered PSMA-RADS-3A lesions to be lymph node or soft tissue findings that had subtle radiotracer uptake (approximately blood pool or slightly higher) and that were in a typical pattern of distribution for $\mathrm{PCa}$, such as the pelvis and retroperitoneum $[7,8]$. Additionally, radiotracer uptake in the mediastinum and left supraclavicular space in patients with more advanced disease was considered as typical sites of PCa spread. Lesions that appeared more conspicuous with PSF were re-categorized as PSMA-RADS-4: a category that signifies distinct uptake in un-enlarged lymph nodes or other morphologically normal soft tissue structures and that is highly suggestive of the presence of PCa.

For those patients for whom it was available, follow-up cross-sectional imaging was reviewed in order to establish which PSMA-RADS-3A lesions developed into true positive sites of disease. No specific limitations were set on the type of follow-up imaging that could be used, and imaging included repeat ${ }^{18} \mathrm{~F}$-DCFPyL PET/CT, ${ }^{18} \mathrm{~F}$-fluciclovine PET/CT, CT, or MRI. Lesions that were determined on follow-up to be suggestive of the presence of $\mathrm{PCa}$ met at least one of the following criteria, adapted from [9]:

1. Follow-up PET/CT imaging with ${ }^{18} \mathrm{~F}-\mathrm{DCFP} \mathrm{L}$ with significantly increasing or decreasing uptake (defined as a change of $30 \%$ from baseline) after therapy OR significantly increased uptake during observation. If the follow-up PET/CT was performed with ${ }^{18} \mathrm{~F}$-fluciclovine, focal uptake in a lesion in concordance with the read paradigm [14] for that agent was taken as evidence of true positivity.

2. Anatomic imaging with $\mathrm{CT}$ or MRI demonstrating a $\geq 2 \mathrm{~mm}$ increase in the long axis diameter of the lesion during a period of observation or a decrease in the lesion long axis diameter of $\geq 2 \mathrm{~mm}$ after beginning PCa treatment.

In addition to PSMA-RADS version 1.0 categorization, the $\mathrm{SUV}_{\max }$ corrected for lean body mass for all of the lesions and the $S U V_{\text {mean }}$ of blood pool (determined by a $3 \mathrm{~cm}$ sphere in the ascending aorta) were measured. The ratios of $S U V_{\max }$ of each lesion corrected for the $\mathrm{SUV}_{\text {mean }}$ of blood pool (SUV $\mathrm{Sux}_{\text {max }}$-lesion/SUV $\mathrm{S}_{\text {mean }}$-blood-pool) were calculated.

\subsection{Statistical Analysis}

Descriptive statistics were utilized for this post hoc analysis. Patient demographic and clinical information are given as medians with ranges or percentages, as appropriate.

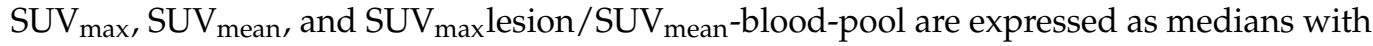
interquartile ranges (IQRs). Median values were compared using the Mann-Whitney U test with $p$-values $<0.05$ considered significant. Statistical analysis was performed with PASW Statistics 17.0 (Chicago, IL, USA).

\section{Results}

\subsection{Patients}

Clinical and imaging parameters of 275 patients were screened. Of those, 89 patients were categorized as having at least one PSMA-RADS-3A lesion on standard clinical reconstructions of their ${ }^{18} \mathrm{~F}-\mathrm{DCFPyL}$ PET/CT scans. Thirty of those patients were found to (1) have been imaged on the Siemens Biograph mCT scanner with PSF reconstruction images of their ${ }^{18} \mathrm{~F}$-DCPFyL PET/CTs available in the picture archiving and communica- 
tion system (PACS) and (2) had adequate follow-up cross-sectional imaging to evaluate for potential changes over time in the PSMA-RADS-3A lesions.

Those 30 patients were included in the presented analysis. Their median age was 72.5 (range 59-81) and median serum PSA at the time of imaging was 3.8 (range 0.2-43.9). Twenty-two (73.3\%) underwent imaging for biochemical recurrence or PSA persistence following local therapy, 4 (13.3\%) underwent imaging for initial PCa staging, and $4(13.3 \%)$ underwent imaging for evaluation of metastatic disease. With regard to available follow-up imaging, $20(66.7 \%)$ underwent diagnostic abdomen/pelvis CT scans, $6(20.0 \%)$ underwent repeat ${ }^{18} \mathrm{~F}$-DCFPyL PET /CT, $3(10.0 \%)$ underwent ${ }^{18} \mathrm{~F}$-fluciclovine PET/CT, and $1(3.3 \%)$ underwent a pelvic MRI. Follow-up imaging was performed at a median of 11 months (range: 2-36 months) post initial ${ }^{18} \mathrm{~F}-\mathrm{DCFPyL}$ PET/CT. Selected demographic and clinical information of the study cohort are included in Table 1.

Table 1. Treatment data from patients included in this study.

\begin{tabular}{|c|c|}
\hline Therapy & Percent \\
\hline $\begin{array}{l}\text { Pre-Scan Therapy } \\
\text { - Prostatectomy } \\
\text { - Radiation } \\
\text { - Salvage Radiation } \\
\text { - Brachytherapy } \\
\text { - Cryoablation } \\
\text { - ADT } \\
\text { - Salvage PLND } \\
\text { - None }\end{array}$ & $\begin{array}{c}70 \% \\
36 \% \\
30 \% \\
3 \% \\
3 \% \\
30 \% \\
3 \% \\
13 \%\end{array}$ \\
\hline $\begin{array}{l}\text { Post-Scan Therapy } \\
\text { - Prostatectomy } \\
\text { - Cryoablation } \\
\text { - Radiation } \\
\text { - ADT } \\
\text { - Salvage PLND } \\
\text { - Chemotherapy } \\
\text { - Observation }\end{array}$ & $\begin{array}{c}7 \% \\
3 \% \\
23 \% \\
60 \% \\
7 \% \\
20 \% \\
13 \%\end{array}$ \\
\hline
\end{tabular}

Abbreviations: ADT = androgen deprivation therapy; PLND = pelvic lymph node dissection.

\subsection{Image Analysis}

Among the 30 patients included in this study, a total of 171 lesions were designated as PSMA-RADS-3A (median 4 per patient, range 1-23), all of which were lymph nodes. Of these lesions, 13/171 (7.6\%) demonstrated visual changes with PSF reconstructions such that they were re-categorized as PSMA-RADS-4 (Figure 1). A total of 112/171 (65.5\%) lesions were found on follow-up imaging to be indicative of PCa, 32/112 $(28.6 \%)$ by category 1 above, and 80/112 (71.4\%) by category 2 above. A total of 13/13 (100.0\%) of the re-categorized lesions were found to be true positive on follow-up imaging, whereas $99 / 158(62.7 \%)$ lesions that were still considered indeterminate on the PSF reconstructions were found to be true positive on follow-up.

Uptake characteristics of lesions are summarized in Figures 2 and 3. The analyses

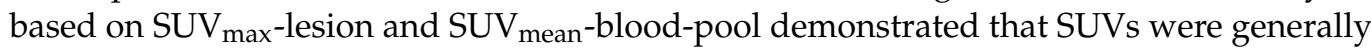

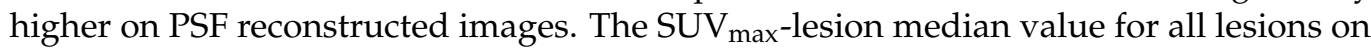
non-PSF images was 1.7 (IQR 1.2-2.1). The SUV $\max$-lesion median value all lesions on PSF

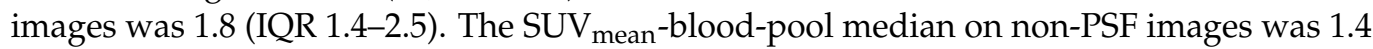
(IQR 1.3-1.5) and on PSF images was 1.4 (IQR 1.3-1.6). SUV max $_{\text {-lesion/SUV }}$ mean-blood-pool median for all lesions on non-PSF images was 1.1 (IQR 0.8-1.6). SUV $\mathrm{Sax}_{\text {-lesion/SUV }}$ mean $^{-}$ blood-pool for all lesions on PSF images was 1.3 (IQR 0.9-1.9).

For the 13 PSMA-RADS-3A lesions that were re-categorized to PSMA-RADS-4, the

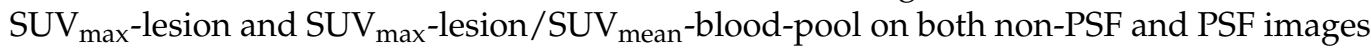
were generally higher than those lesions that were not re-categorized (Figure 2), although 
these differences were not statistically significant $(p=0.085-0.216)$. Similar to our prior findings [9], there was no difference in the $\mathrm{SUV}_{\max }$-lesion and $\mathrm{SUV}_{\max }-\mathrm{lesion} / \mathrm{SUV}_{\text {mean- }}$ blood-pool values between those lesions that were true positive and those that were not confirmed to be true positive (Figure 2, $p=0.198-0.811$ ). The distributions of uptake in the different lesion categories overlapped on both non-PSF and PSF reconstructed images (Figure 3).
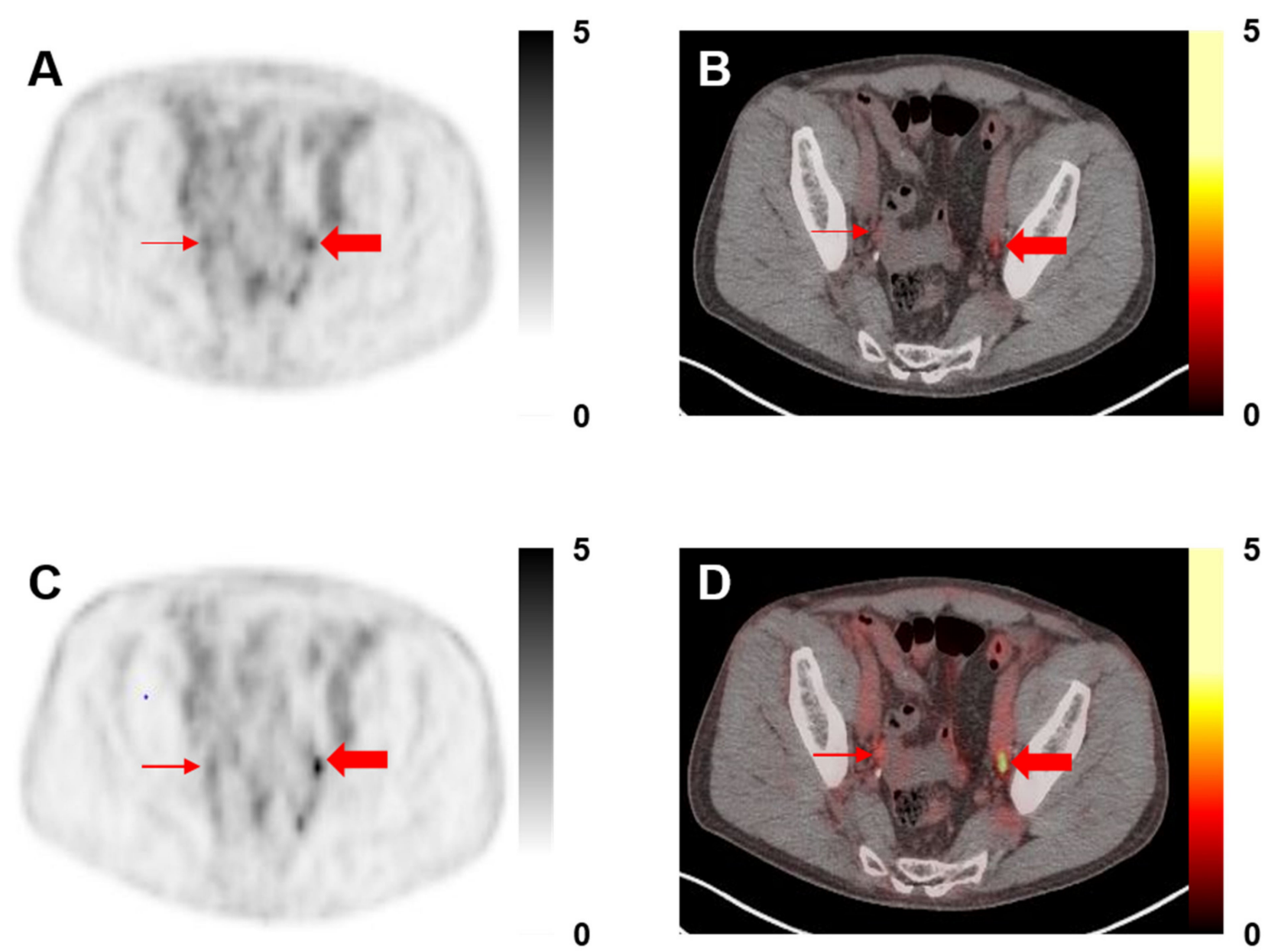

Figure 1. A 68-year-old man with history of prostatectomy and adjuvant chemohormonal therapy, now with an increase in prostate-specific antigen (PSA) to 0.3. (A) ${ }^{18} \mathrm{~F}-\mathrm{DCFPyL}$ positron emission tomography (PET) and (B) PET/computed tomography (CT) axial images without point-spread function (PSF) demonstrating bilateral PSMA-RADS-3A pelvic lymph nodes (thin and thick red arrows, maximum standardized uptake value $\left(\mathrm{SUV}_{\max }\right) 1.2$ on the right and 1.5 on the left). (C) ${ }^{18}$ F-DCFPyL PET and (D) PET/CT axial images with PSF show that the right pelvic PSMA-RADS-3A lymph node does not appear significantly more conspicuous $\left(\mathrm{SUV}_{\max } 2.2\right)$ and that lesion was not re-categorized. However, the lymph node on the left $\left(\mathrm{SUV}_{\max } 2.5\right)$ was thought to be more conspicuous and was re-categorized to PSMA-RADS-4. 


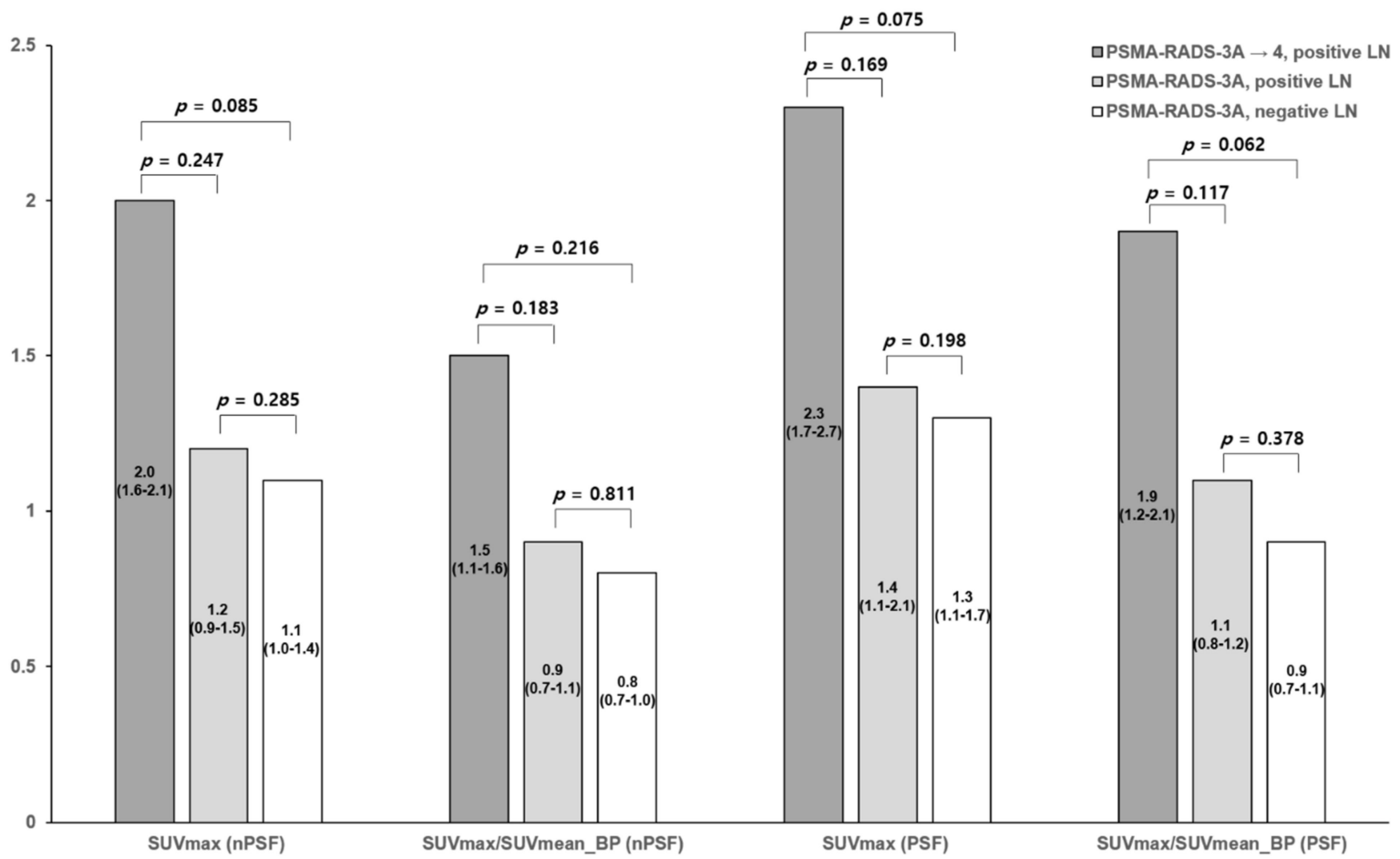

Figure 2. Bar graph representation of medians and interquartile ranges for the $S U_{\text {max }}-l_{\text {lesion }}$ and $S U V_{\text {max }}-l_{\text {lesion }} / S_{U V} V_{\text {mean }}{ }^{-}$ blood-pool metrics of the PSMA-RADS-3A lesions included in this study. In each bar of the graph, the top number is the median and the interquartile ranges are listed below. Note the trends towards higher SUVs of the lesions that were re-categorized as PSMA-RADS-4 (dark grey), although none of these relationships reached statistical significance (i.e., all $p$ > 0.05). SUV = standardized uptake value; $\mathrm{nPSF}=$ non-point-spread function; $\mathrm{BP}=$ blood-pool; $\mathrm{PSF}=$ point-spread function; $\mathrm{LN}=$ lymph node.

SUVmax (nPSF)

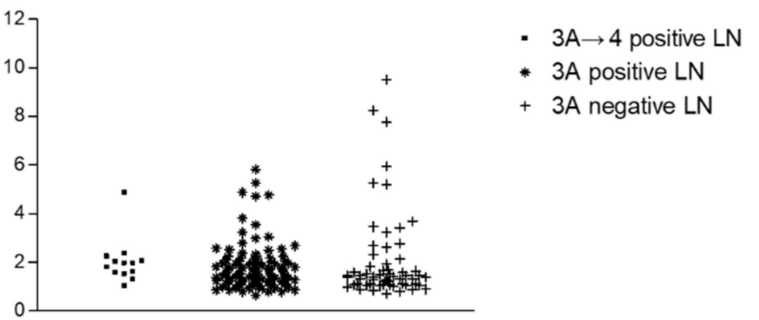

SUVmax/SUVmean_BP (nPSF)

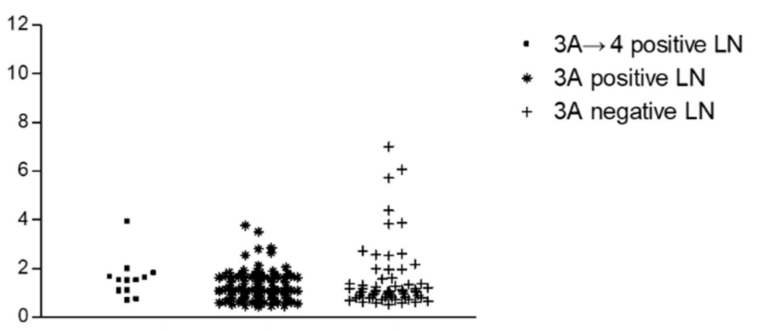

SUVmax (PSF)

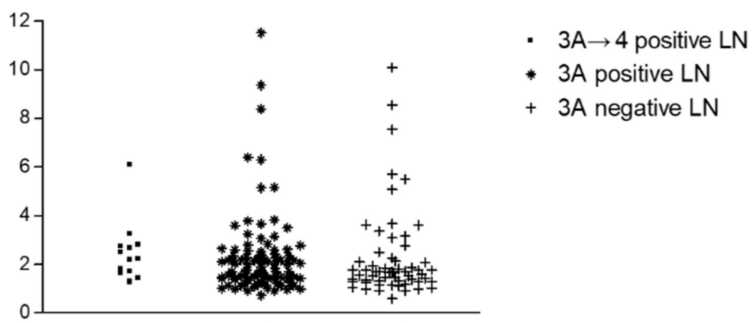

SUVmax/SUVmean_BP (PSF)

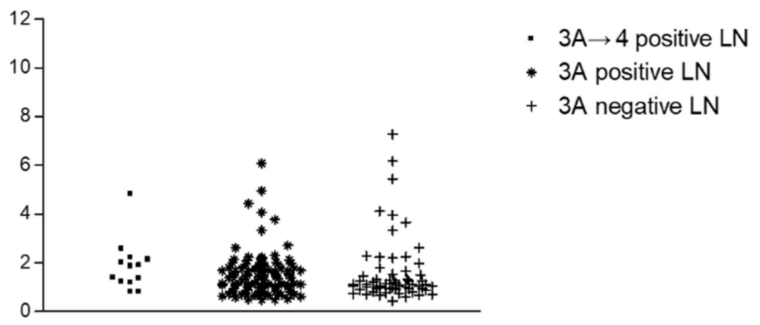

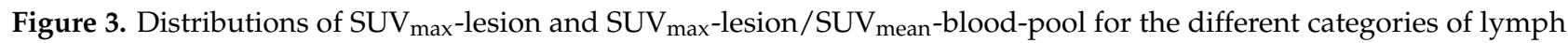
nodes included in this study. The outlier high uptake values were related to overlap of other high uptake structures in the manual segmentations of the lesions. SUV = standardized uptake value; $\mathrm{nPSF}=$ non-point-spread function; $\mathrm{BP}=\mathrm{blood}$-pool; PSF = point-spread function; $\mathrm{LN}=$ lymph node. 


\section{Discussion}

PSMA-targeted PET has been rapidly adopted around the world for PCa imaging given its high sensitivity and specificity for the identification of sites of disease $[15,16]$. However, as with any imaging modality, there are indeterminate findings that belie easy categorization [17]. The preponderance of studies on PSMA-targeted PET imaging has been retrospective, further hindering the ability of readers to know how best to guide management of indeterminate findings, although large prospective studies are starting to appear in the literature [18].

In this study, PSF reconstruction methods for ${ }^{18} \mathrm{~F}$-DCFPyL PET/CT scans were evaluated in terms of characterization of indeterminate PSMA-RADS-3A lesions. We found that PSF reconstructions can increase the contrast and activity concentration within PSMARADS-3A lesions, and that based on a visual analysis, there was a subset of lesions that were changed to the more definitive category of PSMA-RADS-4 (7.6\%). Of those lesions that were determined to change to PSMA-RADS-4 on the PSF-reconstructed images, all were determined to be true positive on follow-up imaging. This suggests that PSF reconstructions can increase confidence in the characterization of a small number of indeterminate lesions on PSMA-targeted PET scans, and that confidence does not come at the cost of decreased specificity. It should be noted that the lesions that were re-categorized tended to have higher $\mathrm{SUV}_{\max }$-lesion and $\mathrm{SUV}_{\max }-$ lesion/SUV $\mathrm{Sean}_{\text {-blood-pool than those lesions }}$ that were not re-categorized (although the trend did not reach significance), suggesting that careful segmentation and determination of semi-quantitative parameters may aid in PSMA-RADS classification and that it may not be optimized as a purely visual method of lesion categorization.

Regardless of reconstruction methodology, the vast majority of indeterminate soft tissue lesions on PSMA PET will remain indeterminate. As we have previously shown [9], and consistent with the present work, a sizable majority of indeterminate soft tissue lesions will be true positive on follow-up ( $65.5 \%$ in this study, similar to the $75.0 \%$ reported in [9]), and it may be prudent to include such lesions in the planning of salvage therapies, such as stereotactic body radiation therapy for oligometastatic disease [19]. A subset of lesions that could not be confirmed as true positive may still represent sites of PCa; however, they did not undergo the changes that we had designated a priori as indicative of true positive lesions. It is likely that artificial intelligence algorithms will be able to leverage the large amounts of scan data to eventually aid in the definitive characterization of otherwise indeterminate findings [20].

An important limitation of this study is the lack of histopathologic confirmation of PSMA-RADS-3A lesions. Such lesions on ${ }^{18}$ F-DCFPyL PET scans are often smaller than $1 \mathrm{~cm}$, making biopsy impossible to perform. Thus, we were required to rely upon imaging follow-up to define a true positive lesion. Other limitations of our study include its post hoc design and small sample size of only 30 patients. Nonetheless, a large number of lesions were analyzed allowing for confidence in our findings. Further, the central review that was utilized was primarily driven by one reader with a second, corroborating reader; this could have introduced bias. Lastly, for small lesions with subtle uptake, the physical differences in image quality between ${ }^{18} \mathrm{~F}$ and ${ }^{68} \mathrm{Ga}$ may be relevant, and it is uncertain if the findings in this study would directly apply to ${ }^{68} \mathrm{Ga}$-labeled PSMA agents. Certainly, given these limitations, further study of this topic is warranted.

\section{Conclusions}

We evaluated the impact of PSF reconstruction for ${ }^{18} \mathrm{~F}-\mathrm{DCFPyL}$ PET $/ \mathrm{CT}$ on indeterminate PSMA-RADS-3A lesions in patients with PCa. A small number of lesions became more definitively characterized with the addition of PSF, and all of those that were re-categorized were found to be true positive on follow-up. We conclude that PSF reconstructions for PSMA-targeted PET may appropriately increase confidence in the characterization of a small, yet clinically significant, number of otherwise indeterminate lesions. 
Author Contributions: W.K. collected data and wrote the manuscript. H.W.C. collected data, performed statistical analyses, and revised the manuscript. R.A.W. collected data and revised the manuscript. J.P.L. provided information technology support, collected data, and revised the manuscript. K.J.P. provided supervision and funding and revised the manuscript. M.A.L. performed data analysis, provided supervision, and revised the manuscript. M.A.G. provided supervision and revised the manuscript. M.G.P. provided supervision and funding and revised the manuscript. S.P.R. conceived the study, wrote the manuscript, and provided supervision and funding. All authors have read and agreed to the published version of the manuscript.

Funding: We acknowledge funding from CA134675, CA183031, CA184228, EB024495, the Prostate Cancer Foundation Young Investigator Award, and Progenics Pharmaceuticals.

Institutional Review Board Statement: The study was conducted according to the guidelines of the Declaration of Helsinki, and approved by the Institutional Review Board of Johns Hopkins Hospitals (protocol code IRB00084971, approved 25 May 2016).

Informed Consent Statement: Informed consent was obtained from all subjects involved in the study.

Data Availability Statement: The data presented in this study are available on request from the corresponding author. The data are not publicly available due to privacy limitations.

Conflicts of Interest: M.G.P. is a coinventor on a U.S. patent covering ${ }^{18} \mathrm{~F}-\mathrm{DCFPyL}$ and as such is entitled to a portion of any licensing fees and royalties generated by this technology. This arrangement has been reviewed and approved by the Johns Hopkins University in accordance with its conflictof-interest policies. S.P.R. is a consultant to Progenics Pharmaceuticals, the licensee of ${ }^{18} \mathrm{~F}-\mathrm{DCFPyL}$. M.A.G. has served as a consultant to Progenics Pharmaceuticals. K.J.P., M.A.G., M.G.P. and S.P.R. receive research funding from Progenics Pharmaceuticals.

\section{References}

1. Siegel, R.L.; Miller, K.D.; Jemal, A. Cancer statistics, 2018. CA Cancer J. Clin. 2018, 68, 7-30. [CrossRef] [PubMed]

2. Murphy, D.G.; Sweeney, C.J.; Tombal, B. “Gotta catch 'em all”, or do we? Pokemet approach to metastatic prostate cancer. Eur. Urol. 2017, 72, 1-3. [CrossRef] [PubMed]

3. Perner, S.; Hofer, M.D.; Kim, R.; Shah, R.B.; Li, H.; Möller, P.; Hautmann, R.E.; Gschwend, J.E.; Kuefer, R.; Rubin, M.A. Prostatespecific membrane antigen expression as a predictor of prostate cancer progression. Hum. Pathol. 2007, 38, 696-701. [CrossRef] [PubMed]

4. $\quad$ Rowe, S.P.; Gorin, M.A.; Allaf, M.E.; Pienta, K.J.; Tran, P.T.; Pomper, M.G.; Ross, A.E.; Cho, S.Y. PET imaging of prostate-specific membrane antigen in prostate cancer: Current state of the art and future challenges. Prostate Cancer Prostatic Dis. 2016, 19, 223-230. [CrossRef] [PubMed]

5. Reyes, D.K.; Pienta, K.J. The biology and treatment of oligometastatic cancer. Oncotarget 2015, 6, 8491-8524. [CrossRef] [PubMed]

6. Cornford, P.; Bellmunt, J.; Bolla, M.; Briers, E.; de Santis, M.; Gross, T.; Henry, A.M.; Joniau, S.; Lam, T.B.; Mason, M.D.; et al. EAU-ESTRO-SIOG Guidelines on Prostate Cancer. Part II: Treatment of Relapsing, Metastatic, and Castration-Resistant Prostate Cancer. Eur. Urol. 2017, 71, 630-642. [CrossRef] [PubMed]

7. Rowe, S.P.; Pienta, K.J.; Pomper, M.G.; Gorin, M.A. Proposal for a structured reporting system for prostate-specific membrane antigen-targeted PET imaging: PSMA-RADS version 1.0. J. Nucl. Med. 2018, 59, 479-485. [CrossRef] [PubMed]

8. Rowe, S.P.; Pienta, K.J.; Pomper, M.G.; Gorin, M.A. PSMA-RADS version 1.0: A step towards standardizing the interpretation and reporting of PSMA-targeted PET imaging studies. Eur. Urol. 2018, 73, 485-487. [CrossRef] [PubMed]

9. Yin, Y.; Werner, R.A.; Higuchi, T.; Lapa, C.; Pienta, K.J.; Pomper, M.G.; Gorin, M.A.; Rowe, S.P. Follow-up of lesions with equivocal radiotracer uptake on PSMA-targeted PET in patients with prostate cancer: Predictive values of the PSMA-RADS-3A and PSMA-RADS-3B categories. J. Nucl. Med. 2019, 60, 511-516. [CrossRef] [PubMed]

10. Werner, R.A.; Bundschuh, R.A.; Bundschuh, L.; Javadi, M.S.; Leal, J.P.; Higuchi, T.; Pienta, K.J.; Buck, A.K.; Pomper, M.G.; Gorin, M.A.; et al. Interobserver agreement for the standardized reporting system PSMA-RADS 1.0 on ${ }^{18} \mathrm{~F}-\mathrm{DCFPyL}$ PET/CT imaging. J. Nucl. Med. 2018, 59, 1857-1864. [CrossRef] [PubMed]

11. Andersen, F.L.; Klausen, T.L.; Loft, A.; Beyer, T.; Holm, S. Clinical evaluation of PET image reconstruction using a spatial resolution model. Eur. J. Radiol. 2013, 82, 862-869.

12. Ravert, H.T.; Holt, D.P.; Chen, Y.; Mease, R.C.; Fan, H.; Pomper, M.G.; Dannals, R.F. An improved synthesis of the radiolabeled prostate-specific membrane antigen inhibitor, [18F]DCFPyL. J. Labelled Comp. Radiopharm. 2016, 59, 439-450. [CrossRef] [PubMed]

13. Rowe, S.P.; Gorin, M.A.; Hammers, H.J.; Javadi, M.S.; Hawasli, H.; Szabo, Z.; Cho, S.Y.; Pomper, M.G.; Allaf, M.E. Imaging of metastatic clear cell renal cell carcinoma with PSMA-targeted 18F-DCFPyL PET/CT. Ann. Nucl. Med. 2015, 29, 877-882. [CrossRef] [PubMed]

14. Schuster, D.M. Axumin ${ }^{\mathrm{TM}}$ (Fluciclovine F 18) Image Interpretation Training. Available online: https:/ / www.snmmilearningcenter. org/lms/activity?@curriculum.id=-1\&@activity.id=4521746\&@activity.bundleActivityId=-1 (accessed on 10 February 2021). 
15. Perera, M.; Papa, N.; Christidis, D.; Wetherell, D.; Hofman, M.S.; Murphy, D.G.; Bolton, D.; Lawrentschuk, N. Sensitivity, specificity, and predictors of positive 68Ga-prostate-specific membrane antigen positron emission tomography in advanced prostate cancer: A systematic review and meta-analysis. Eur. Urol. 2016, 70, 926-937. [CrossRef] [PubMed]

16. Murphy, D.G.; Hofman, M.; Lawrentschuk, N.; Maurer, T. Bringing clarity or confusion? The role of prostate-specific membrane antigen positron-emission/computed tomography for primary staging in prostate cancer. BJU Int. 2017, 119, 194-195. [CrossRef] [PubMed]

17. Sheikhbahaei, S.; Afshar-Oromieh, A.; Eiber, M.; Solnes, L.B.; Javadi, M.S.; Ross, A.E.; Pienta, K.J.; Allaf, M.E.; Haberkorn, U.; Pomper, M.G.; et al. Pearls and pitfalls in clinical interpretation of prostate-specific membrane antigen (PSMA)-targeted PET imaging. Eur. J. Nucl. Med. Mol. Imaging 2017, 44, 2117-2136. [CrossRef] [PubMed]

18. Hofman, M.S.; Lawrentschuk, N.; Francis, R.J.; Tang, C.; Vela, I.; Thomas, P.; Rutherford, N.; Martin, J.M.; Frydenberg, M.; Shakher, R.; et al. Prostate-specific membrane antigen PET-CT in patients with high-risk prostate cancer before curative intent surgery or radiotherapy (proPSMA): A prospective, randomized, multicentre study. Lancet 2020, 395, 1208-1216. [CrossRef]

19. Phillips, R.; Shi, W.Y.; Deek, M.; Radwan, N.; Lim, S.J.; Antonarakis, E.S.; Rowe, S.P.; Ross, A.E.; Gorin, M.A.; Deville, C.; et al. Outcomes of observation vs. stereotactic ablative radiation for oligometastatic prostate cancer: The ORIOLE phase 2 randomized clinical trial. JAMA Oncol. 2020, 6, 650-659. [CrossRef] [PubMed]

20. Moazemi, S.; Khurshid, Z.; Erle, A.; Lütje, S.; Essler, M.; Schultz, T.; Bundschuh, R.A. Machine learning facilitates hotspot classificiation in PSMA-PET/CT with nuclear medicine specialist accuracy. Diagnostics 2020, 10, 622. [CrossRef] [PubMed] 